\section{New Theeory}

ISSN: 2149-1402
37 (2021) 99-107

Journal of New Theory

https://dergipark.org.tr/en/pub/jnt

Open Access

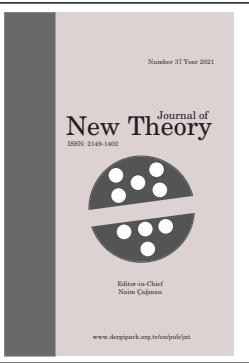

\title{
Generating Generalized Cylinder with Geodesic Base Curve According to Darboux Frame
}

\author{
Nabil Mohammed Althibany ${ }^{1}$ (D)
}

\author{
Article History \\ Received: 14 Dec 2021 \\ Accepted: 29 Dec 2021 \\ Published: 31 Dec 2021 \\ 10.53570/jnt.1036307 \\ Research Article
}

\begin{abstract}
This paper aims to design a generalized cylinder with a geodesic base curve according to the Darboux frame in Euclidean 3-space. A generalized cylinder is a special ruled surface that is constructed by a continuous fixed motion of a generator line called the ruling along a given curve called the base curve. The necessary and sufficient conditions for the base curve to be geodesic are studied. The main results show that the generalized cylinder with a geodesic base curve is an osculating cylinder whose base curve is a helical geodesic, and the rulings are directed by the unit osculating Darboux vector.
\end{abstract}

Keywords - Generalized cylinder, geodesic, Darboux frame, general helix, osculating Darboux vector

Mathematics Subject Classification (2020) - 53A04, 53A05

\section{Introduction}

A generalized cylinder is constructed by a constant motion of a straight line called the ruling through a given curve called the base curve. The generalized cylinders are a class of developable ruled surfaces that have no singularities points and can be produced from paper or sheet metal with no distortion. For this construction, the generalized cylinder has been investigated as a basic modeling surface in various fields of science including geometric modeling, computer graphic, architectural designing [1-4].

Geodesic is a characteristic curve that is used to obtain the shortest distance between two points on curved space. Geodesic is a curve that travels through the surface such that its principal normal vector field parallel to the surface normal vector field. The different spaces and surfaces are characterized by their geodesics, the lines and grate circles are geodesics on the plane and the sphere respectively, but the (meridian) circles are not always geodesics on the surface of revolution. The helices are geodesics on the cylinder but the helicoid contains helices that are not geodesics [5-8]. Calculating and visualizing the geodesics on different spaces are important problems in many areas of applications [9-11].

A helical curve (or a helix) is a space curve in Euclidean 3-space whose tangent vector field makes a constant angle with a constant direction. According to the Theorem of Lancret [12], a necessary and sufficient condition that a curve is of constant slope (or general helix ) is that the ratio of torsion to curvature is constant. The circular helix is a special helix with both its curvature and torsion are constants, based on this definition, the circle and line are degenerate helices [13]. A circular cylinder contains a helix, a circle, and a line as geodesics. The relation between generalized cylinder, geodesic, and a helix is well known and can be summarized by "a helix is a geodesic on a general cylinder".

A helical geodesic is a curve in which its principal normal vector field parallel to the surface normal vector field and at the same time its tangent vector makes a constant angle with a fixed direction.

\footnotetext{
${ }^{1}$ althibany1972@yahoo.com

${ }^{1}$ Department of Mathematics, Faculty of Applied Science, Taiz University, Taiz, Yemen
} 
Thus, a helical geodesic is characterized by both two geometric natures, a helix from the Euclidean 3 -space viewpoint, and a geodesic from the ambient surface viewpoint, for more details, see $[5,8,14]$.

Darboux frame is adapted moving frame defined along a curve that lives on the surface. It is an important tool to study the geometry of surface curves such as geodesic curves. Darboux frame is defined at each point of the surface curve and can be obtained by rotating the Frenet frame around the tangent vector. The Darboux and Frenet frames agree modulo signs along the geodesic curves [15].

An osculating Darboux vector is defined by Izumiya and Otani [16] as the special direction of the Darboux frame that lies in the tangent plane of the surface. They showed that an osculating Darboux vector field has a constant direction if and only if the osculating developable surface is a generalized cylinder. An osculating developable surface is a ruled surface whose rulings are directed by the osculating Darboux vector field along the curve, it is tangent to the surface along the curve and it gives a flat approximation of the surface along the curve [17]. An osculating Darboux vector was studied in $[18,19]$, it characterizes the helical geodesic on the osculating cylinder as shown in this paper.

Designing the surface or the surfaces family that possess the given curve as a geodesic curve has been studied by Wang et al. [20], where they derived a sufficient condition for the curve to be geodesic on a given surface. After that, Kasap et al. [21] generalized the work of Wang. A developable surface that possesses a base curve as a geodesic has been studied in [22]. Recently in [23], the author studied and classified the ruled surfaces whose base curve is characteristic. After that in [24], we concluded that among all developable surfaces, the generalized cylinder can be equipped with geodesic coordinates if and only if the base curve is a helix and the director vector is a unit Darboux vector.

The main goal of this paper is to design a generalized cylinder whose base curve is geodesic in Euclidean 3- space. The generalized cylinders are a class of ruled surfaces, therefore, we start from a ruled parametrization, then with additional conditions called the cylindrical conditions, we define the generalized cylinder parametrization according to the Darboux frame. After that, and under some geometric constraints, we obtain the base curve as a geodesic curve. The main results show that the generalized cylinder with the geodesic base curve is an osculating cylinder in which the base curve is a helical geodesic and the rulings are directed by a constant unit osculating Darboux vector.

The rest of this paper is organized as follows: In Section 2, some basic notations, facts, and definitions of space curve in Euclidean 3-space and on a surface are reviewed. The main results are studied in Section 3, where the generalized cylinder with a geodesic base curve is generated. Finally, the conclusion is given in Section 4.

\section{Preliminary}

This section introduces some basic concepts on the classical differential geometry of the curve lying on the surface and in Euclidean 3-space, as well as some basic definitions and notions that are required subsequently. More details can be found in such standard references as $[12,25,26]$.

\subsection{Curves in Euclidean 3-space}

A smooth space curve in 3-dimensional Euclidean space is parameterized by a map $\gamma: I \subseteq \mathbb{R} \rightarrow E^{3}$, $\gamma$ is called a regular curve if $\gamma^{\prime} \neq 0$ for every point of an interval $I \subseteq \mathbb{R}$, and if $\left|\gamma^{\prime}(s)\right|=1$ where $\left|\gamma^{\prime}(s)\right|=\sqrt{\left\langle\gamma^{\prime}(s), \gamma^{\prime}(s)\right\rangle}$, then $\gamma$ is said to be of unit speed (or parameterized by arc-length $s$ ). For a unit speed regular curve $\gamma(s)$ in $E^{3}$, the unit tangent vector $t(s)$ of $\gamma$ at $\gamma(s)$ is given by $t(s)=\gamma^{\prime}(s)$. If $\gamma^{\prime \prime}(s) \neq 0$, the unit principal normal vector $\mathrm{n}(\mathrm{s})$ of the curve at $\gamma(s)$ is given by $n(s)=\frac{\gamma^{\prime \prime}(s)}{\left\|\gamma^{\prime \prime}\right\|}$. The unit vector $b(s)=t(s) \times n(s)$ is called the unit binormal vector of $\gamma$ at $\gamma(s)$. For each point of $\gamma(s)$ where $\gamma^{\prime \prime}(s) \neq 0$, we associate the Serret-Frenet frame $\{t, n, b\}$ along the curve $\gamma$. As the parameter $\mathrm{s}$ traces out the curve, the Serret-Frenet frame moves and satisfies the following Frenet-Serret formula.

$$
\left\{\begin{array}{l}
t^{\prime}(s)=\kappa(s) n(s), \\
n^{\prime}(s)=-\kappa(s) t(s)+\tau b(s), \\
b^{\prime}(s)=-\tau(s) n(s),
\end{array}\right.
$$


where $\kappa=\kappa(s)$ and $\tau=\tau(s)$ are the curvature and torsion functions. When the point moves along the unit speed curve with non vanishing curvature and torision, the Serret-Frenet frame $\{t, n, b\}$ is drawn to the curve at each position of the moving point, this motion consists of translation with rotation and described by the following Darboux vector whose direction is the direction of rotational axis and its magnitude gives the angular velocity of rotation.

$$
\omega=\tau t+\kappa b
$$

The general helix $\left(\left(\frac{\tau}{\kappa}=c\right)\right)$ lies on a general cylinder and also known as a cylindrical helix. The circular helix ( a helix on a circular cylinder) is a special helix with both of $\kappa(s) \neq 0$ and $\tau(s)$ are constants. The Darboux vector is constant for circular helix. For the cylindrical helix, the unit Darboux vector is constant as following

$$
\hat{\omega}=\frac{\tau}{\sqrt{\tau^{2}+\kappa^{2}}} t+\frac{\kappa}{\sqrt{\tau^{2}+\kappa^{2}}} b=\frac{\tau / \kappa}{\sqrt{(\tau / \kappa)^{2}+1}} t+\frac{1}{\sqrt{(\tau / \kappa)^{2}+1}} b=\frac{c}{\sqrt{c^{2}+1}} t+\frac{1}{\sqrt{c^{2}+1}} b
$$

For a regular curve on a surface, there exists another frame called Darboux frame and denoted by $\{t(s), g(s), N(s)\}$, where $t(s)$ is the unit tangent of the curve, $N(s)$ is the unit normal of the surface and $g$ is a unit vector given by $g=N \times t$. Derivative of the Darboux frame is given by the following

$$
\left\{\begin{array}{l}
t^{\prime}(s)=\kappa_{g} g(s)+\kappa_{n} N(s), \\
g^{\prime}(s)=-\kappa_{g} t(s)+\tau_{g} N(s), \\
N^{\prime}(s)=-\kappa_{n} t(s)-\tau_{g} g(s),
\end{array}\right.
$$

where $\kappa_{g}$ is the geodesic curvature, $\kappa_{n}$ is the normal curvature and $\tau_{g}$ is the geodesic torsion at each point of the curve $\gamma(s)$ which are given by

$$
\kappa_{g}=<\gamma^{\prime \prime}(s), g>, \quad \kappa_{n}=<\gamma^{\prime \prime}(s), N>\text {, and } \quad \tau_{g}=<N^{\prime}, g>
$$

The relations between Frenet and Darboux frames can be given by the following matrix form

$$
\left(\begin{array}{c}
t \\
g \\
N
\end{array}\right)=\left(\begin{array}{ccc}
1 & 0 & 0 \\
0 & \cos \phi & \sin \phi \\
0 & -\sin \phi & \cos \phi
\end{array}\right)\left(\begin{array}{l}
t \\
n \\
b
\end{array}\right)
$$

where

$$
\left\{\begin{array}{l}
g(s)=\cos \phi(s) n(s)+\sin \phi(s) b(s), \\
N(s)=-\sin \phi(s) n(s)+\cos \phi(s) b(s)
\end{array}\right.
$$

Differentiating (7) using (4) and (1), we get the relation between geodesic curvature, normal curvature, and geodesic torsion with curvature and torsion as follows:

$$
\kappa_{g}=\kappa \cos \phi, \quad \kappa_{n}=\kappa \sin \phi, \quad \tau_{g}=\tau+\frac{d \phi}{d s}, \quad \text { where } \kappa^{2}(s)=\kappa_{n}^{2}(s)+\kappa_{g}^{2}(s)
$$

Definition 2.1. A unit-speed curve on a surface is called a geodesic if and only if its geodesic curvature is zero $\left(\kappa_{g}=0\right)$.

Remark 2.2. For a geodesic curve $\left(\kappa_{g}=0\right)$, some geometric quantities along the geodesic curve are congruent up to orientation, the principal normal of the curve and the surface normal are parallel to each other at any point on the curve, the rectifying plane coincides with the tangent plane of the surface, the Frenet and Darboux frames agree modulo signs, the absolute value of the normal curvature and geodesic torsion equal to the ordinary curvature and torsion respectively. By using (7) and (8), the following equations can be calculated easily

$$
N= \pm n, \quad g= \pm b, \quad \kappa_{n}= \pm \kappa, \text { and } \quad \tau_{g}= \pm \tau
$$




\section{Generalized Cylinder with Geodesic Base Curve}

This section is the main part of the present paper and it consists of three subsections. In the first subsection we give the necessary and sufficient conditions for the ruled parametrization to be generalized cylinder parametrization. The second subsection is devoted to making the base curve of the generalized cylinder is a geodesic. In the third subsection, we prove that the geodesic base curve is a helix, or equivalently a helical geodesic. Th conditions which are assumed are given by Darboux frame and its related geometric quantities. The main result is obtained subsequently and shows that the generalized cylinder with the geodesic base curve is an osculating cylinder whose base curve is a helical geodesic and the rulings are directed by a constant unit osculating Darboux vector.

\subsection{Generalized Cylinder}

A generalized cylinder is generated by a constant moving of a straight line on a given curve and defined by the following ruled parametrization

$$
X(s, v)=\gamma(s)+v D(s), 0 \leq s \leq \ell, v \in \mathbb{R}, \text { where } D^{\prime}(s)=0
$$

A unit regular curve $\gamma(s)$ is called the base curve, the line passing through $\gamma(s)$ that is parallel to $D(s)$ is called the ruling. $D(s)$ is a unit director vector field that gives the direction of the ruling, $D^{\prime}(s)=0$ is the cylindrical condition which means that the ruling moves in a constant direction. $D(s)$ lies in the tangent plane of the generalized cylinder and can be written using (6) as following

$$
D(s)=\cos \theta(s) t(s)+\sin \theta(s) g(s)
$$

The derivative of $D(s)$ in terms of the Darboux frame and its derivatives is given by

$$
D^{\prime}(s)=-\sin \theta(s)\left[\kappa_{g}(s)+\frac{d \theta}{d s}\right] t(s)+\cos \theta(s)\left[\kappa_{g}(s)+\frac{d \theta}{d s}\right] g(s)+\left[\kappa_{n}(s) \cos \theta(s)+\tau_{g}(s) \sin \theta(s)\right] N(s)
$$

Definition 3.1. The ruled parametrization with base curve $\gamma(s)$ and a unit director vector $\mathrm{D}(\mathrm{s})$ is defined by

$$
\begin{cases}X(s, v) & =\gamma(s)+v D(s), 0 \leq s \leq L, v \in \mathbb{R}, \text { where } \\ D(s) & =\cos \theta(s) t(s)+\sin \theta(s) g(s) .\end{cases}
$$

The following Theorem gives the cylindrical conditions that make the ruled parametrization (13) to be generalized cylinder parametrization

Theorem 3.2. The ruled parametrization (13) is a generalized cylinder if and only if the following conditions are satisfied

$$
\begin{aligned}
\kappa_{g}(s)+\frac{d \theta}{d s} & =0, \\
\kappa_{n}(s) \cos \theta(s)+\tau_{g}(s) \sin \theta(s) & =0
\end{aligned}
$$

Proof. By definition, the ruled parametrization (13) is a cylinder if and only if $D^{\prime}(s)$ vanishes, by using (12) this condition is satisfied provided that (14) are satisfied.

Definition 3.3. The generalized cylinder with base curve $\gamma(s)$ and a unit director vector D(s) (11) is parameterized by

$$
\left\{\begin{array}{l}
X(s, v)=\gamma(s)+v[\cos \theta(s) t(s)+\sin \theta(s) g(s))], 0 \leq s \leq L, v \in \mathbb{R}, \quad \text { where } \\
\kappa_{g}(s)+\frac{d \theta}{d s}=0 \quad \text { and } \quad \kappa_{n}(s) \cos \theta(s)+\tau_{g}(s) \sin \theta(s)=0
\end{array}\right.
$$

The main result of this paper is the following main theorem which is proved in the next section.

Theorem 3.4. Let $X(s, v)=\gamma(s)+v D(s), 0 \leq s \leq L, v \in \mathbb{R}$ be a generalized cylinder, $\gamma(s)$ is a unit speed regular curve with non vanishing curvature and torsion, $D(s)$ is a unit director vector defined by (11) satisfying $D^{\prime}(s)=0$. Then the generalized cylinder with geodesic base curve is an osculating cylinder whose base curve is a helical geodesic and $D(s)$ is a constant unit osculating Darboux vector. 


\subsection{Generalized Cylinder with Geodesic Base Curve}

This subsection is devoted to constructing a generalized cylinder parameterized by (15) with the geodesic base curve. For this purpose, three conditions must be satisfied as given in the following

Theorem 3.5. A base curve $\gamma(s)$ of the generalized cylinder parameterized by (15) is a geodesic if and only if the following conditions are satisfied

$$
\begin{aligned}
\kappa_{g}(s) & =0, \\
\frac{d \theta}{d s} & =0, \\
\kappa_{n}(s) \cos \theta(s)+\tau_{g}(s) \sin \theta(s) & =0
\end{aligned}
$$

Proof. By definition (2.1), a base curve $\gamma(s)$ of the generalized cylinder parameterized by (15) is a geodesic if and only if $\kappa_{g}=0$ which is the first condition of (16). By substitution it in the cylindrical conditions (14), we get the other conditions of (16).

Definition 3.6. A generalized cylinder with geodesic base curve is defined by

$$
\left\{\begin{array}{l}
X(s, v)=\gamma(s)+v[\cos \theta(s) t(s)+\sin \theta(s) g(s)], 0 \leq s \leq L, v \in \mathbb{R}, \quad \text { where } \\
\kappa_{g}(s)=0, \frac{d \theta}{d s}=0, \text { and } \quad \kappa_{n}(s) \cos \theta(s)+\tau_{g}(s) \sin \theta(s)=0
\end{array}\right.
$$

Izumiya and Otani [16] defined the osculating Darboux vector field $D_{0}$ and its normalized $\hat{D}_{0}$ provided $\left(\kappa_{n}(s), \kappa_{g}(s)\right) \neq(0,0)$ as the following

$$
D_{0}=\tau_{g}(s) t(s)-\kappa_{n}(s) g(s), \quad \hat{D}_{0}=\frac{\tau_{g}(s)}{\sqrt{\tau_{g}^{2}(s)+\kappa_{n}^{2}(s)}} t-\frac{\kappa_{n}(s)}{\sqrt{\tau_{g}^{2}(s)+\kappa_{n}^{2}(s)}} g
$$

Proposition 3.7. Suppose that $D(s)=\cos \theta(s) t(s)+\sin \theta(s) g(s)$ is a unit vector field defined along a unit speed surface curve $\gamma(s)$ with $\left(\tau_{g}(s), \kappa_{n}(s)\right) \neq(0,0)$, then $D(s)$ is a unit osculating Darboux vector field if and only if $\kappa_{n}(s) \cos \theta(s)+\tau_{g}(s) \sin \theta(s)=0$.

Proof. Let $D(s)=\cos \theta(s) t(s)+\sin \theta(s) g(s)$ be unit osculating Darboux vector field. From (18)

$$
\cos \theta=\frac{\tau_{g}(s)}{\sqrt{\tau_{g}^{2}(s)+\kappa_{n}^{2}(s)}}, \quad \sin \theta(s)=\frac{-\kappa_{n}(s)}{\sqrt{\tau_{g}^{2}(s)+\kappa_{n}^{2}(s)}}, \quad \text { and } \quad \cot \theta=-\frac{\tau_{g}(s)}{\kappa_{n}(s)}
$$

This implies that $\kappa_{n}(s) \cos \theta(s)+\tau_{g}(s) \sin \theta(s)=0$, and vice versa.

The condition $\frac{d \theta}{d s}=0$ implies that the angle function $\theta(s)$ is constant, hence the rulings keep constant angle to a fixed direction $D(s)$, or equivalently $D^{\prime}(s)=0$ as a cylindrical condition. This condition with proposition (3.7) insure that the unit osculating Darboux vector field is constant.

Theorem 3.8. A base curve $\gamma(s)$ of the generalized cylinder parameterized by (15) is a geodesic if and only if $D(s)$ is a constant unit osculating Darboux vector.

Definition 3.9. A generalized cylinder with geodesic base curve is defined by

$$
\begin{cases}X(s, v) & =\gamma(s)+v D(s), 0 \leq s \leq L, v \in \mathbb{R} \quad \text { where } \\ D(s) & =\frac{\tau_{g}(s)}{\sqrt{\tau_{g}^{2}(s)+\kappa_{n}^{2}(s)}} t(s)-\frac{\kappa_{n}(s)}{\sqrt{\tau_{g}^{2}(s)+\kappa_{n}^{2}(s)}} g(s), \quad D^{\prime}(s)=0\end{cases}
$$




\subsection{Generalized Cylinder with Helical Geodesic Base Curve}

In the last subsection, Theorem (3.8) and definition (3.9) presented the conditions that can be applied on the director vector field $D(s)$ to make the base curve of the generalized cylinder to be geodesic. Therefore, this subsection investigates the equivalent conditions that can be applied on the base curve to be geodesic. For this purpose, we start by the following theorem, for proof see [27].

Theorem 3.10. [27] A unit speed curve on a surface with $\left(\kappa_{n}(s), \kappa_{g}(s)\right) \neq(0,0)$ is a general helix if and only if

$$
\frac{1}{\left(\kappa_{n}^{2}(s)+\kappa_{g}^{2}(s)\right)^{3 / 2}}\left(\kappa_{g}^{\prime} \kappa_{n}-\kappa_{n}^{\prime} \kappa_{g}-\tau_{g}\left(\kappa_{n}^{2}+\kappa_{g}^{2}\right)\right)=\text { constant }
$$

For a geodesic curve, $\kappa_{g}(s)=0$, the condition (20) is rewritten as the following

Corollary 3.11. Let $\gamma(s)$ be a geodesic curve with $\kappa_{n}(s) \neq 0$. Then, $\gamma(s)$ is a helix if and only if

$$
\frac{\tau_{g}(s)}{\kappa_{n}(s)}=\text { constant }
$$

Under the assumption that $\kappa_{n}(s) \neq 0$, and by substitution (21) in (18), the following corollary determines the relationship between a helix, geodesic curve, and the unit osculating Darboux vector.

Corollary 3.12. Let $\gamma(s)$ be a geodesic curve on a surface with $\kappa_{n}(s) \neq 0$. Then, $\gamma(s)$ is a helix if and only if the unit osculating Darboux vector field $\hat{D}_{0}$ is a constant.

The unit osculating Darboux is a vector on the surface that plays a role analogous to the unit Darboux vector (3) in $E^{3}$, they characterize the helix on the surface and $E^{3}$ respectively, compare proposition (3.7) and corollary (3.12) with their counterparts in [23,24]. The following theorem gives the satisfying conditions on the base curve and director vector to make a base curve is a geodesic.

Theorem 3.13. A base curve $\gamma(s)$ of the generalized cylinder parameterized by (15) is a geodesic if and only if $\gamma(s)$ is a helix and $D(s)$ is a unit osculating Darboux vector.

Definition 3.14. A generalized cylinder with geodesic base curve is defined by

$$
\left\{\begin{array}{l}
X(s, v)=\gamma(s)+v D(s), 0 \leq s \leq L, v \in \mathbb{R}, \quad \text { where } \\
D(s)=\frac{\tau_{g}(s)}{\sqrt{\tau_{g}^{2}(s)+\kappa_{n}^{2}(s)}} t(s)-\frac{\kappa_{n}(s)}{\sqrt{\tau_{g}^{2}(s)+\kappa_{n}^{2}(s)}} g(s), \quad \text { and } \gamma(s) \text { is a helix. }
\end{array}\right.
$$

Theorem 3.15. Let $X(s, v)=\gamma(s)+v D(s), 0 \leq s \leq L, v \in \mathbb{R}$ be a ruled surface, where $\gamma(s)$ is a unit speed regular surface curve with $\left(\tau_{g}(s), \kappa_{n}(s)\right) \neq(0,0), D(s)$ is a unit direction vector defined by $D(s)=\cos \theta(s) t(s)+\sin \theta(s) g(s)$. Then $\mathrm{X}(\mathrm{s}, \mathrm{v})$ is a generalized cylinder whose base curve is a geodesic if and only if $\gamma(s)$ is a helix and $D(s)$ is a unit osculating Darboux vector.

A ruled surface whose rulings are directed by the osculating Darboux vector along the curve is called an osculating developable surface [16]. The generalized cylinder defined by (22) is a special case where the unit osculating Darboux vector is a constant and we call it the osculating cylinder.

Theorem 3.16. Let $X(s, v)=\gamma(s)+v D(s), 0 \leq s \leq L, v \in \mathbb{R}$ be a generalized cylinder, where $\gamma(s)$ is a unit speed regular surface curve with $\left(\tau_{g}(s), \kappa_{n}(s)\right) \neq(0,0)$, and $D(s)=\cos \theta(s) t(s)+\sin \theta(s) g(s)$ satisfies $D^{\prime}(s)=0$. Then the following properties are equivalent:

i. $\mathrm{X}(\mathrm{s}, \mathrm{v})$ is an osculating cylinder,

ii. $D(s)$ is a constant unit osculating Darboux vector,

iii. $\gamma(s)$ is a geodesic,

iv. $\gamma(s)$ is a helix. 
Theorem 3.17. Among all generalized cylinders parameterized by (15), only the osculating cylinder (22) has a geodesic base curve.

The approximation of a given surface by a flat surface (developable) along the arbitrary curve is called flat or developable approximation. An osculating developable is tangent to the surface along a curve and it gives a flat approximation of the surface along the curve [17]. The osculating cylinder (22) inherits this property and gives flat approximation of the surface along a helical geodesic. Approximating by an osculating cylinder (22) has nice geometrical properties: globally free of singularities, achieved along a geodesic path, some geometric quantities on both cylinder and the surface along the geodesic curve are congruent up to orientation (2.2), locally like approximating by the ribbon [28], and the cylinder is easy to modeling and reasoning from a mathematical or manufacturing viewpoint. For more details about the flat approximations of surfaces and hypersurfaces see [16, 17, 29].

\section{Conclusion}

The work in this paper can be separated into two parts as given subsequently in the third section. Firstly, we presented how the generalized cylinder (15) can be parameterized from a ruled parametrization (13) under the cylindrical conditions, see Theorem (3.2) and definition (3.3). Secondly, we showed how the generalized cylinder with geodesic base curve is generated, see Theorem (3.13) and definition (3.14). The generated cylinder is an osculating (22) whose base curve is a helical geodesic and the director vector is a unit osculating Darboux vector. Among all generalized cylinders (15), only the osculating cylinder (22) has geodesic base curve. The unit osculating Darboux vector can be used as a tool not only to generate the osculating cylinder (22), but to characterize the helical geodesic, corollary (3.12). The osculating cylinder (22) has nice geometrical properties, one of them it gives a flat approximation of the surface along a helical geodesic.

\section{Author Contributions}

The author read and approved the last version of the manuscript.

\section{Conflicts of Interest}

The author declares no conflict of interest.

\section{References}

[1] K. H. Chang, Product Design Modeling Using CAD/CAE, The Computer Aided Engineering Design Series, Academic Press, 2014.

[2] R. Goldman, An Integrated Introduction to Computer Graphics and Geometric Modeling, CRC Press, 2009.

[3] S. Guha, Computer Graphics through OpenGL: From Theory to Experiments, Chapman and Hall/CRC, 2018.

[4] H. Pottmann, A. Asperl, M. Hofer, A. Kilian, Architectural Geometry, Bentley Institute Press, Exton, 2007.

[5] M. Tamura, Surfaces Which Contain Helical Geodesics, Geometriae Dedicata 42(3) (1992) 311315.

[6] A. Görgülü, Surfaces Which Contain Inclined Curves as Geodesics, Communications Faculty of Sciences University of Ankara Series A1 Mathematics and Statistics 42 (1993) 39-44.

[7] M. Tamura, Surfaces Which Contain Helical Geodesics in the 3-Sphere, Memoirs of the Faculty of Science and Engineering Shimane University. Series B. Mathematical Science 37 (2004) 59-65. 
[8] D. W. Yoon, On Constructions of Minimal Surfaces, Journal of the Chungcheong Mathematical Society 34(1) (2021) 1-15.

[9] I. Hotz, H. Hagen, Visualizing Geodesics, In Proceedings Visualization VIS 2000 (Cat. No. 00CH37145) IEEE (2000) 311-318.

[10] G. R. Kumar, P. Srinivasan, V. D. Holla, K. G. Shastry, B. G. Prakash, Geodesic Curve Computations on Surfaces, Computer Aided Geometric Design 20(2) (2003) 119-133.

[11] E. Kasap, M. Yapici, F. T. Akyildiz, A Numerical Study for Computation of Geodesic Curves, Applied Mathematics and Computation 171(2) (2005) 1206-1213.

[12] D. J. Struik, Lectures on Classical Differential Geometry, 2nd Edition, Dover Publications Inc., New York, 1988.

[13] A. T. Ali, New Special Curves and Their Spherical Indicatrix, Global Journal of Advanced Research On Classical and Modern Geometries 1(2) (2012) 28-38.

[14] R. Lopez, G. Ruiz-Hernández, A Characterization of Isoparametric Surfaces in $\mathbb{R}^{3}$ Via Normal Surfaces, Results in Mathematics 67(1) (2015) 87-94.

[15] M. Huard, N. Sprynski, N. Szafran, L. Biard, Reconstruction of Quasi Developable Surfaces from Ribbon Curves, Numerical Algorithms 63(3) ( 2013) 483-506.

[16] S. Izumiya, S. Otani, Flat Approximations of Surfaces Along Curves, Demonstratio Mathematica 48(2) (2015) 217-241.

[17] S. I. Honda, S. Izumiya M. Takahashi, Developable Surfaces Along Frontal Curves on Embedded Surfaces, Journal of Geometry 110(2) (2019) 1-20.

[18] S. Hananoi, N. Ito, S. Izumiya, Spherical Darboux Images of Curves on Surfaces, Beiträge zur Algebra und Geometrie 56(2) (2015) 575-585.

[19] S. Izumiya, K. Saji, N. Takeuchi, Flat Surfaces Along Cuspidal Edges, Journal of Singularities 16 (2017) $73-100$.

[20] G. J. Wang, K. Tang, C.L. Tai, Parametric Representation of a Surface Pencil with a Common Spatial Geodesic, Computer-Aided Design 36(5) (2004) 447-459.

[21] E. Kasap, F.T. Akyildiz, K. Orbay, A generalization of surfaces family with common spatial geodesic, Applied Mathematics and Computation 201(1-2) (2008) 781-789.

[22] R. A. Al-Ghefaria, A. B. Rashad, An Approach for Designing a Developable Surface with a Common Geodesic Curve, International Journal of Contemporary Mathematical Sciences 8(18) (2013) 875-891.

[23] N. M. Althibany, Classification of Ruled Surfaces Family with Common Characteristic Curve in Euclidean 3-space, Turkish Journal of Science 6(2) (2021) 61-70.

[24] N. M. Althibany, Construction of Developable Surface with Geodesic or Line of Curvature Coordinates, Journal of New Theory (36) (2021) 75-87.

[25] M. D. Carmo, Differential Geometry of Curves and Surfaces, Prentice-Hall, New Jersey, 1976.

[26] A. N. Pressley, Elementary Differential Geometry, Springer Science \& Business Media, 2010.

[27] M. Düldül, B. U. Düldül, Characterizations of helices by using their Darboux Vectors, Sigma: Journal of Engineering \& Natural Sciences 38(3) (2020) 1299-1306. 
[28] M. Raffaelli, J. Bohr, S. Markvorsen, Cartan Ribbonization and a Topological Inspection, Proceedings of the Royal Society A 474(2220) (2018) p.20170389.

[29] I. Markina, M. Raffaelli, Flat Approximations of Hypersurfaces Along Curves, Manuscripta Mathematica 160(3) (2019) 315-325. 